\title{
Interpretación batimétrica del área de Valparaíso: comentarios sobre el artículo de HP Vergara \& R Astudillo, 'Altos estructurales costa afuera en la región de Valparaíso, Chile central'
}

Bathymetric interpretation of the Valparaiso area: comments on the paper by HP Vergara \& R Astudillo, 'Offshore structural highs of the Valparaiso region, central Chile’

\section{Cristián Rodrigo ${ }^{1}$}

${ }^{1}$ Departamento Científico, Instituto Antártico Chileno, Plaza Muñoz Gamero 1055, Punta Arenas, Chile. crodrigo@inach.cl

\begin{abstract}
A submarine alignment at 320-33으 offshore within the Valparaiso Region, Chile, is composed by three peaks at 200, 733 and $880 \mathrm{~m}$ below sea level, which were identified by Vergara \& Astudillo (2008) using a bathymetric public data base. This is composed by bathymetry which is estimated by satellite altimetry, and from ship surveys. However, after modeling recent similar data bases and also processing and modeling available multibeam bathymetry with $\sim 100 \%$ of spatial coverage, those highs seem to be absent. The apparent creation of bathymetric artifacts would have been due to the inclusion of bad single beam data of a specific ships sounding track. The hypothesis regarding the origin of the alignment associated with the vertical displacement of a block at the outer shelf, would not be valid.
\end{abstract}

Key words: Satellite altimetry, multibeam bathymetry, continental margin, seafloor features

\section{INTRODUCCIÓN}

A pesar de la importancia del conocimiento de los fondos marinos para la caracterización y aprovechamiento de los recursos naturales, la navegación y muchas otras aplicaciones para aumentar la calidad de vida de los seres humanos, se han confeccionado mejores mapas de planetas, lunas y asteroides que del fondo del mar, el cual permanece desconocido en un $90 \%$ a un minuto de arco de resolución (Becker et al. 2009). Esto es debido a las grandes dimensiones de los océanos, las limitaciones tecnológicas y logísticas para realizar extensos sondajes batimétricos y las restricciones de los métodos de teledetección por satélites. Sin embargo, un gran avance para el conocimiento de los fondos marinos, fue la creación de un modelo topográfico que consideró la utilización de datos batimétricos derivados de altimetría satelital y datos batimétricos provenientes de ecosondas de buques (Smith \& Sandwell 1997, Sandwell \& Smith 2001), que permitió determinar finalmente, todas las estructuras del fondo del mar a escala intermedia y global, logrando definir bien cuerpos mayores de $25 \mathrm{~km}$. Varios controles de calidad fueron efectuados a los datos satelitales y también para aquellos de buques, sin embargo, estos últimos provenían de diversas fuentes que no aplicaban procedimientos homogéneos de corrección, por ejemplo, las de Smith (1993). La base de datos batimétrica original de 1997 se ha ido mejorando a través del tiempo al incluir más datos de buques y aumentar la resolución (e.g., Becker et al. 2009).

Vergara \& Astudillo (2008) utilizaron la base de datos de topografía del Laboratorio de Geodesia Satelital de Scripps Institution of Oceanography (SIO), correspondiente a la SRTM (Shuttle Radar Topography Mission) ${ }^{1}$, para construir un modelo topográfico submarino frente a la región de Valparaíso, Chile, entre los $32^{\circ}$ y $33^{\circ} \mathrm{S}$. Su interpretación indica la presencia de un lineamiento submarino compuesto de tres cumbres a 200, 733 y $880 \mathrm{~m}$ de profundidad, orientado $\mathrm{N} 10^{\circ} \mathrm{W}$. Sostienen que su origen puede estar relacionado con procesos tectónicos tensionales asociados a la zona de ruptura de la placa oceánica descendente y su interacción con la placa superior, que produjeron un levantamiento de un bloque no confinado del borde externo de la plataforma.

${ }^{1}$ http:// www.jpl. nasa.gov/ srtm 
En el talud medio de esta área se localiza la terraza Valparaíso a $\sim 2500 \mathrm{~m}$ de profundidad, donde subyace la cuenca antearco Valparaíso. La cuenca se dispone a lo largo del eje N-S (60 por $40 \mathrm{~km}$ ) y tiene un espesor de sedimentos de entre 3000 a 3500 m (von Huene et al. 1997). La formación de esta cuenca ha sido atribuida a dos procesos principales controlados e influenciados por la subducción de la dorsal o cadena de Juan Fernández (Yáñez et al. 2001, Laursen et al. 2002): erosión basal de la placa continental a lo largo de la parte somera y asísmica de la interfase de subducción; y la deformación compresional de la parte frontal del margen continental, la cual es inducida por la subducción de montes marinos y cordones de la dorsal de Juan Fernández (Laursen \& Normark 2003). El resultado de estos procesos es una rotación del talud superior y la plataforma que, progresivamente, va formando una cuenca profunda submarina bajo el talud medio que se deforma en su lado occidental, en altos estructurales de antearco (basamento) (von Huene et al. 1997, Yáñez et al. 2001, Laursen \& Normark 2003). Por el lado sur $\left(\sim 33^{\circ} \mathrm{S}\right)$, la cuenca es confinada por el montículo Topocalma, el cual podría marcar la localización de un monte marino subducido (Laursen \& Normark 2002); y por el lado norte, se limita por el cordón Punta Salinas, que se originaría por la subducción de parte de la cadena montes marinos de Juan Fernández (von Huene et al. 1997, Yáñez et al. 2001). Por lo tanto, dada las características tectónicas y morfológicas especiales del área, Vergara \& Astudillo (2008) generaron un modelo topográfico submarino utilizando datos batimétricos recientes y encontraron nuevos altos estructurales en el sector del talud superior, explicándolas como resultado de esta compleja tectónica.

Sin embargo, la siguiente discusión muestra que los datos batimétricos utilizados por Vergara \& Astudillo (2008) para generar el modelo topográfico del área, carecían de un control de calidad completo que permitiera identificar la totalidad de los datos erróneos o dudosos, por lo cual fueron considerados datos de mala calidad, creándose artificios batimétricos e interpretados como altos estructurales del fondo marino, a pesar de la existencia de antecedentes científicos anteriores que no mostraban esos rasgos. El objetivo de esta nota es demostrar que algunas bases públicas de datos científicos, como la utilizada por Vergara \& Astudillo (2008), contienen errores y que su utilización sin correcciones podrían llevar a realizar interpretaciones geológicas y/o tectónicas equivocadas; además de identificar la fuente del error del modelo batimétrico confeccionado por los citados autores.

\section{Materiales y MÉTOdos}

La base de datos utilizada por Vergara \& Astudillo (2008) consiste de datos topográficos terrestres obtenidos por la SRTM y datos de topografía submarina que incluye la batimetría derivada de altimetría satelital (Smith \& Sandwell 1997) y la obtenida por sondajes de buques de las siguientes instituciones principales: Lamont-Doherty Earth Observatory (Columbia University), National Geophysical Data Center (NGDC) y SIO, entre otras. La malla (grid) digital de esta base de datos tiene una resolución de 30 s de $\operatorname{arco}(\sim 1 \mathrm{~km})$, aunque para la parte submarina se basa en la de Smith \& Sandwell (1997) de 1 min.

Para comparar con el modelo de Vergara \& Astudillo (2008), se confeccionaron 4 modelos o mallas digitales independientes utilizándose las mismas características en resolución (30 s) y método de interpolación (superficie continua con tensión ajustable; Smith \& Wessel 1990), con las siguientes bases de datos: SRTM utilizada por Vergara \& Astudillo (2008); la SRTM Plus que es la actualización a noviembre de 2009 de la anterior (Becker et al. 2009); Smith \& Sandwell (1997) actualizada a diciembre de 2009 (http: //topex.ucsd.edu); y de batimetría multihaz Hydrosweep obtenida del crucero CONDOR, a bordo del R/V 'Sonne' en 1995 (von Huene et al. 1995, Vergara 1996, von Huene et al. 1997), que incluye contornos batimétricos de la General Bathymetric Chart of the Oceans (GEBCO) como referencia para la parte costera. La información batimétrica multihaz del crucero CONDOR fue procesada según los procedimientos descritos por von Huene et al. (1995).

También se generaron 2 modelos de la topografía submarina a menor escala para áreas específicas de interés, utilizando sólo datos de batimetría multihaz sin interpolación, para así representar la cobertura espacial real de la batimetría. Para generarlos se utilizaron los datos del crucero CONDOR y del crucero 'Cañón Aconcagua' a bordo del AGOR 'Vidal Gormaz’ en 1996, donde se utilizó el sonar multihaz Sea Beam Plus (Rodrigo 2001), cuyos resultados fueron publicados por SHOA (2002) y por Rodrigo (2010). El procesamiento de los datos de batimetría multihaz Sea Beam Plus consistió en la eliminación de datos mal posicionados, puntas y valores de pendientes exagerados según procedimientos de Caress \& Chayes (2010). Las mallas digitales obtenidas tienen una resolución aproximada de $30 \mathrm{~m}$. Los modelos topográficos correspondientes fueron iluminados para observar mejor las estructuras del fondo marino (con un ángulo de elevación de $30^{\circ}$ y un acimut de $\left.315^{\circ}\right)$. 
Además, para encontrar la posible fuente de error, se generaron otros 2 modelos topográficos considerando sólo datos batimétricos de buques (sondajes) incluidos en la base de datos utilizada por Vergara \& Astudillo (2008), con una resolución aproximada de $500 \mathrm{~m}$. Esto se realizó debido a la observación de la linealidad en la disposición de los altos estructurales, su forma alargada y la coincidencia de su intersección con el puerto de Valparaíso. Para ello se tomaron sólo las trayectorias de buques que tomaron datos en el área de interés. Además, se realizó un análisis de cruces estadístico entre las trayectorias consideradas para determinar el nivel de error de los sondajes según el procedimiento de Wessel (1989).

\section{Resultados y Discusión}

Al contrastar los diferentes mapas del área general (Fig. 1ad) se observaron similitudes en cuanto a la extensión de la plataforma continental y su quiebre, el cual constituye el límite con el talud continental. El talud se extiende hacia el $\mathrm{W}$ desde profundidades mayores a $200 \mathrm{~m}$. En esa zona se aprecia la existencia del cañón submarino Aconcagua, identificado y nombrado por primera vez por von Huene $e t$ al. (1995) y posteriormente, en forma oficial por el Gazetteer de GEBCO de Nombres Geográficos de Rasgos Submarinos en 2003 (OHI-COI 2010) o también informalmente como Montemar (Vergara 1996), el cual es representado en todos los mapas, detallándose mejor su forma en el mapa que utiliza la batimetría del crucero CONDOR (Fig. 1d). Este cañón no está bien representado en el utilizado por Vergara \& Astudillo (2008) y además es el único mapa que muestra el alineamiento de altos estructurales. La posición de la cimas de cada estructura según Vergara \& Astudillo (2008) es mostrada con letras en cada figura. Los demás mapas no muestran ningún alto estructural en las posiciones A y B, encontrándose profundidades de entre 1500 y $1200 \mathrm{~m}$ para el punto A, y de 1200 a 1500 m para el punto B, respectivamente. Sólo para el punto $\mathrm{C}$ se manifiesta un piso marino más elevado, sin embargo, el punto está a una profundidad de entre 700 y $800 \mathrm{~m}$, y no de $200 \mathrm{~m}$. Corresponde considerar que los modelos de las Figs. 1b y 1c representan datos mejorados del mismo modelo de la Fig. 1a. En la base global de datos SRTM Plus (Fig. 1b) fueron editados los datos batimétricos provenientes de buques y se corrigieron alrededor de 298 millones de sondas, disminuyéndose notablemente la creación de artificios batimétricos (Becker et al. 2009).

La información más confiable proviene de la batimetría multihaz cuando se realiza con cobertura completa del fondo del mar y es procesada correctamente. Este es el caso de la batimetría efectuada por el R/V 'Sonne' en el área de estudio. En la Fig. 1d se indica con una línea punteada vertical el límite oriental aproximado del área donde se obtuvo cobertura completa con el sistema de sonar multihaz, por lo cual, no hay duda que las elevaciones en A y B no existen; hecho corroborado por los demás modelos. La batimetría de alta resolución del R/V 'Sonne' ha sido publicada por diversos autores desde el año del crucero en 1995, donde también se observa que al posicionar el lineamiento de altos estructurales en los mapas de esos trabajos, éste no se aprecia (e.g., von Huene et al. 1995, Vergara 1996, von Huene et al. 1997, Yáñez et al. 2001).

Cercano a los puntos A y B mencionados, se encuentran canales submarinos menores indicados con números (Figs. 1d y 1e) e identificados principalmente a partir de los modelos de mayor resolución, cuyas áreas se muestran en el modelo general de la Fig. 1d. Estos canales constituyen depresiones alargadas que posiblemente conduzcan sedimentos desde la plataforma hacia mayores profundidades. Claramente se observa que el punto A se ubica en el borde norte de uno de estos canales $\left(\mathrm{N}^{\circ} 2\right)$ y el $\mathrm{B}$ se encontraría en un punto de intersección entre los canales 3 y 4 (Fig. 1e), considerando la tendencia batimétrica ya que no existió realmente cobertura completa con el sistema multihaz.

Para el caso del punto C, efectivamente en el área existe una elevación que no alcanza los $700 \mathrm{~m}$ de profundidad, la cual parece ser una forma modelada por la erosión de corrientes de turbidez que han socavado el cañón Aconcagua y el canal afluente de más al norte ( $\mathrm{N}^{\circ} 6$, Fig. 1f). El cañón se caracteriza por una serie de meandros o curvas cerca de su cabeza, probablemente debido a que el flujo de sedimentos se desvía al intersectar materiales más competentes creándose diversas formas en el piso marino. Laursen \& Normark (2003), a través del análisis de registros de sísmica de reflexión obtenidos por el R/V 'Sonne' en el crucero CONDOR, explica la formación de elevaciones o salientes en las proximidades del cañón Aconcagua debido a los depósitos de una sucesión de paquetes de sedimentos provenientes del mismo cañón, los cuales se extienden hacia afuera de éste hasta $\sim 25 \mathrm{~km}$. Al conjunto de estas estructuras la llamaron informalmente cordón sedimentario Aconcagua. Con estos antecedentes y a pesar de que se perdió cobertura al utilizar el Sea Beam del AGOR 'Vidal Gormaz', los datos son suficientes para demostrar la inexistencia del alto estructural en el punto C, corroborado también por el sondaje del R/V 'Sonne', el cual navegó precisamente sobre el punto (Fig. 1e). Además, si existieran estos altos estructurales, 

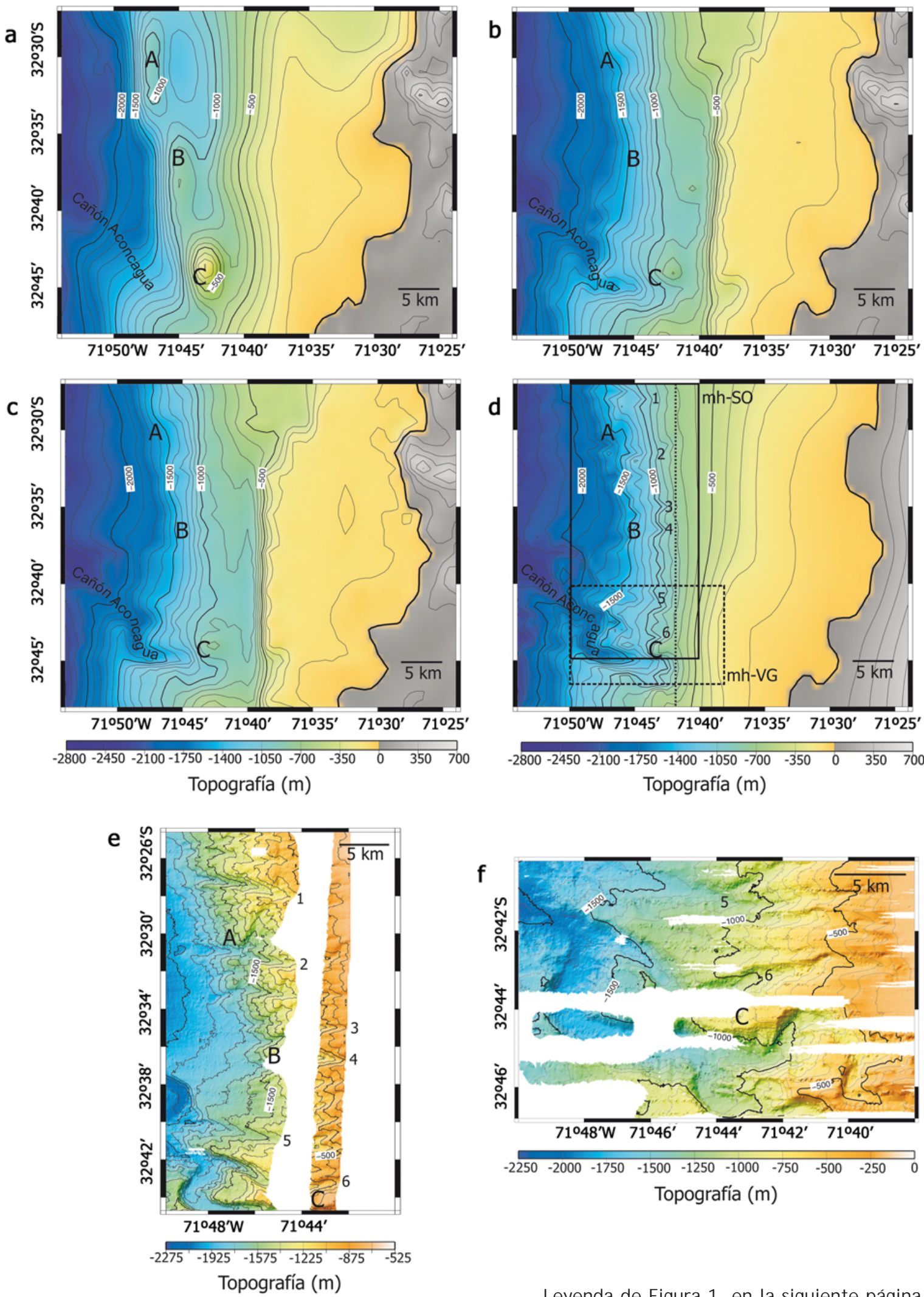

Leyenda de Figura 1, en la siguiente página 
implicaría que el flujo de sedimentos hacia la cuenca estaría interrumpido en el sector donde yacen, lo cual es incongruente con los resultados encontrados por Laursen \& Normark (2003) y los batimétricos de alta resolución presentados.

Como se ha mostrado, las posiciones de las cimas de los supuestos altos estructurales (A, B y C) se encuentran dispuestas en línea. Al crear un nuevo modelo topográfico submarino utilizando sólo los datos batimétricos obtenidos de las trayectorias (tracks) de navegación consideradas en la base de datos SRTM, se identifica el alineamiento que coincide con el mostrado por Vergara \& Astudillo (2008), encontrándose para A y B profundidades de alrededor de $800 \mathrm{~m}$ y para C de $200 \mathrm{~m}$ (Fig. 2a). La posición de cada cima también coincide con un 'track' definido de navegación, el cual es indicado con una línea continua en Fig. 2b. Al realizar el modelo 3D del área se muestra más claramente la serie de elevaciones en línea. Sin embargo, al eliminar los datos de este último 'track' y al confeccionar otro nuevo modelo (Fig. 2d) manteniendo los demás 'track' (Fig. 2e), los altos desaparecen y en su reemplazo se obtienen profundidades similares a las obtenidas por los sistemas multihaz. La Fig. 2f muestra visualmente la desaparición de los altos. El 'track' removido correspondió al ODP141JR, crucero perteneciente al programa Ocean Drilling Program Leg 141, cuyos datos batimétricos fueron obtenidos a bordo del buque R/V ‘Joides Resolution’ en 1992. Además, luego de realizar el análisis estadístico de error entre los cruces de las trayectorias de sondaje, se encontró que el que tenía un mayor nivel de error fue precisamente el crucero
ODP141JR, cuyo RMS fue de 334,9 m, el cual se considera excesivo para las profundidades del área.

En conclusión, los dos altos estructurales septentrionales del área de estudio identificados por Vergara \& Astudillo (2008) no existirían, hecho confirmado por las mediciones de batimetría multihaz efectuadas por el buque R/V ‘Sonne’ en 1995 y los nuevos modelos SRTM Plus y de Smith \& Sandwell actualizados al año 2009. Sobre la elevación en el punto C, ésta tampoco existiría con las características descritas por Vergara \& Astudillo (2008). Probablemente la depresión del mismo cañón Aconcagua y la del afluente de más al norte causen que los modelos tiendan a representar una elevación entre estas depresiones, sumada la existencia de una elevación de origen sedimentario en las proximidades del lado sur del cañón. Se comprueba e identifica que la causa de la aparición de los altos estructurales en el modelo SRTM del año 2007, es la incorporación de datos batimétricos erróneos de tipo monohaz de un sondaje específico efectuado por el buque R/V 'Joides Resolution'.

A pesar de los complejos procesos tectónicos del área de la cuenca Valparaíso, el sector del talud superior ubicado en la porción central de ésta, no mostró evidencias superficiales notables de estos procesos, por lo cual, la hipótesis sobre el origen del alineamiento de altos estructurales asociado al desplazamiento vertical de un bloque en el borde externo de la plataforma, no habría ocurrido.

\begin{abstract}
Figura 1. Mapas de contornos (cada $100 \mathrm{~m}$ ) de la topografía submarina (batimetría) en la región de Valparaíso. A, B y C representan los puntos de las cumbres del lineamiento submarino identificado por Vergara \& Astudillo (2008). Los números indican la posición de canales submarinos menores. a) Base de datos SRTM30 utilizada por Vergara \& Astudillo (2008). b) Base de datos SRTM30 Plus actualizado al año 2009. c) Base de datos de Smith \& Sandwell (1997) actualizado al año 2009. d) Base de datos de batimetría multihaz Hydrosweep del crucero CONDOR de 1995 (al W de línea punteada vertical) y datos de batimetría GEBCO de 1995 (al E de la línea punteada vertical); el cuadro de línea continua (mh-SO) muestra el área de la Fig. 1e; el cuadro de línea segmentada (mh-VG) muestra el área de la Fig. 1f. e) Detalle utilizando batimetría multihaz Hydrosweep del R/V 'Sonne', sin interpolación. f) Detalle utilizando batimetría multihaz Sea Beam Plus del AGOR 'Vidal Gormaz', sin interpolación / Contour submarine topographic (bathymetric) maps of the Valparaiso Region (every $100 \mathrm{~m}$ ). A, B and C represent the peaks of the submarine alignment identified by Vergara \& Astudillo (2008). The numbers indicate the position of smaller submarine channels. a) SRTM30 database used by Vergara \& Astudillo (2008). b) SRTM30 Plus database updated to 2009. c) Smith \& Sandwell (1997) database updated to 2009. d) Hydrosweep multibeam data of the CONDOR cruise, in 1995 (to the W of the dotted vertical line) and GEBCO bathymetry from 1995 (to the E of the dotted vertical line); the box with solid line (mh-SO) shows the area of Fig. le, the box with dashed line (mh-VG) shows the area of Fig. If. e) Detail using the R/V 'Sonne' Hydrosweep multibeam bathymetry, without interpolation. f) Detail using the AGOR 'Vidal Gormaz' Sea Beam Plus multibeam bathymetry, without interpolation
\end{abstract}


a)

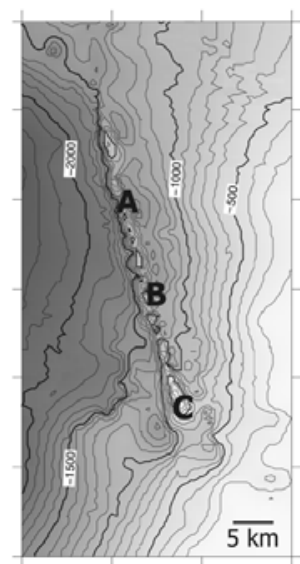

$71^{\circ} 54^{\prime} \mathrm{W} 71^{\circ} 48^{\prime} 71^{\circ} 42^{\prime} 71^{\circ} 36^{\prime}$

d)

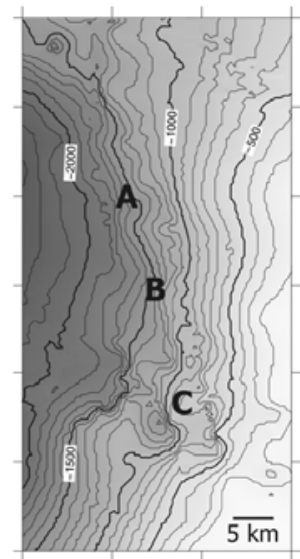

b)

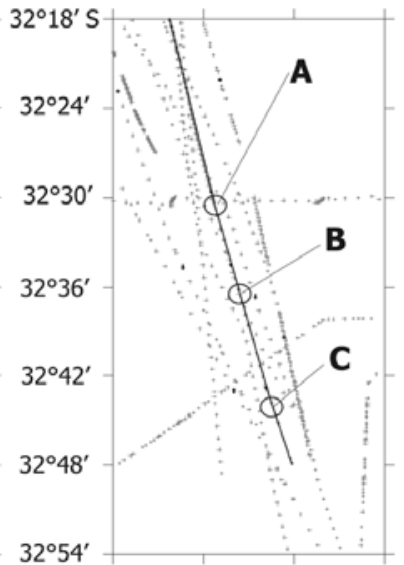

$71^{\circ} 54^{\prime} \mathrm{W} 71^{\circ} 48^{\prime} 71^{\circ} 42^{\prime} 71^{\circ} 36^{\prime}$

e)

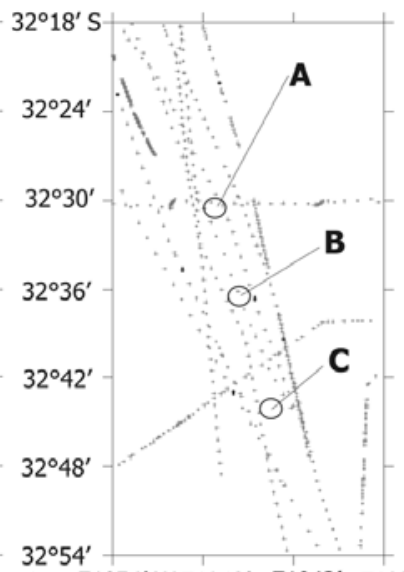

c)
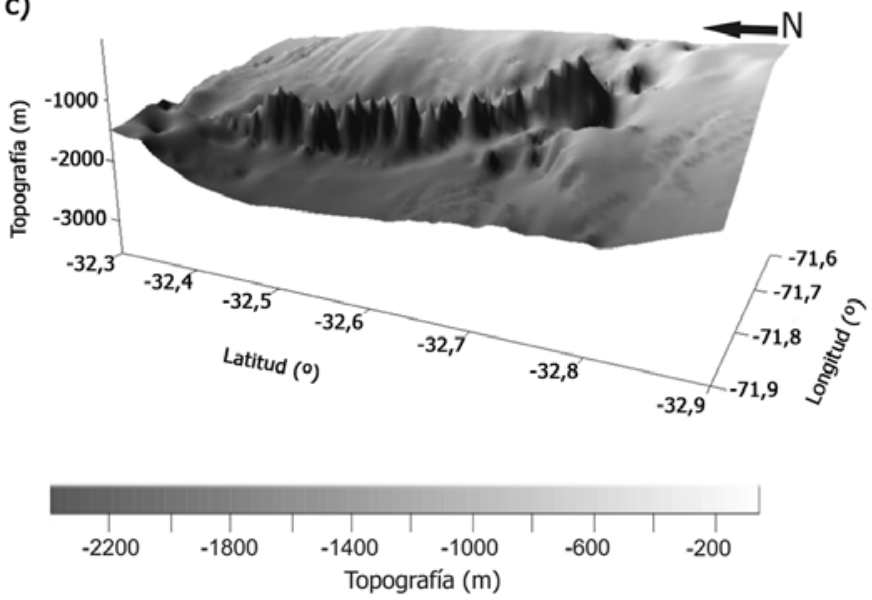

f)

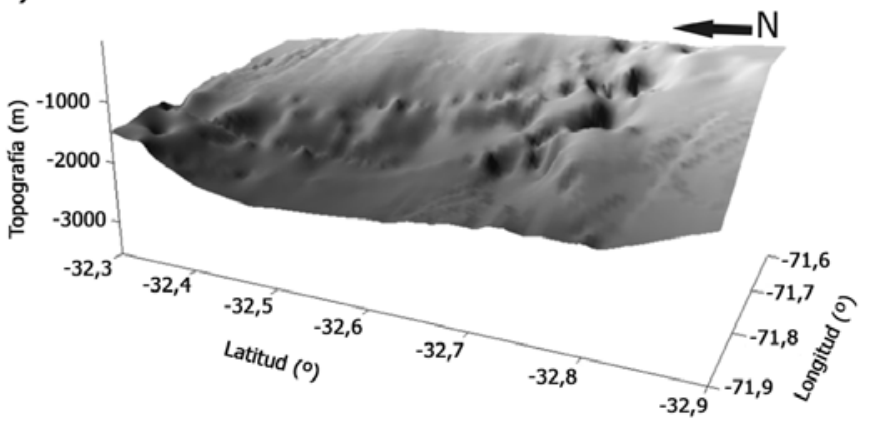

$71^{\circ} 54^{\prime} \mathrm{W} 71^{\circ} 48^{\prime} 71^{\circ} 42^{\prime} 71^{\circ} 36^{\prime} \quad 71^{\circ} 54^{\prime} \mathrm{W} 71^{\circ} 48^{\prime} 71^{\circ} 42^{\prime} 71^{\circ} 36^{\prime}$

Figura 2. Análisis de sondajes que son empleados para la base de datos SRTM30 utilizada por Vergara \& Astudillo (2008). a) Mapa de contornos (cada $100 \mathrm{~m}$ ) de la topografía submarina (batimetría). A, B y C representan los puntos de las cumbres del lineamiento. b) Trayectorias y localización de sondas. La posición de las cumbres se localiza sobre una trayectoria específica (línea continua). c) Modelo 3D utilizando sondas de la Fig. 2b. d) Ídem que Fig. 2a eliminado la trayectoria sobre los puntos A, B y C. e) Ídem que Fig. 2b eliminado la trayectoria sobre los puntos A, B y C. f) Modelo 3D tomando sondas de la Fig. 2e / Analysis of soundings which are used for the SRTM30 data base and used by Vergara \& Astudillo (2008). a) Contour map (every $100 \mathrm{~m}$ ) of the submarine topography (bathymetry). A, B and $\mathrm{C}$ indicate the peaks of the alignment. b) Tracks and sounding location. The peaks are on a specific track (solid line). C) 3D model using soundings of Fig. 2b. d) Same as Fig. 2a eliminating the track at the points A, B and C. e) Same as Fig. 2b eliminating the track at the points A, $B$ and C. f) 3D model using soundings of Fig. 2e 


\section{Agradecimientos}

El autor agradece al Centro Nacional de Datos Hidrográficos y Oceanográficos (CENDHOC), dependiente del Servicio Hidrográfico y Oceanográfico de la Armada (SHOA), por facilitar los datos de batimetría multihaz obtenidos por el R/V 'Sonne' y AGOR 'Vidal Gormaz'. También agradece a los tres revisores anónimos por sus observaciones constructivas que ayudaron a mejorar el trabajo.

\section{LITERATURA CITADA}

Becker JJ, DT Sandwell, WHF Smith, J Braud, B Binder, J Depner, D Fabre, J Factor, S Ingalls, SH Kim, R Ladner, K Marks, S Nelson, A Pharaoh, R Trimmer, J Von Rosenberg, G Wallace \& P Weatherall. 2009. Global bathymetry and elevation data at 30 arc seconds resolution: SRTM30 Plus. Marine Geodesy 32: 355-371.

Caress DW \& DN Chayes. 2010. Mapping the seafloor: software for the processing and display of swath sonar data. [en línea] <http://www.ldeo.columbia.edu/MBSystem/html/mbsystem_home.html>

Laursen J \& WR Normark. 2002. Late Quaternary evolution of the San Antonio Submarine Canyon in the Central Chile

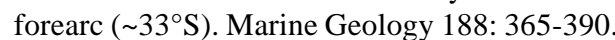

Laursen J \& WR Normark. 2003. Impact of structural and autocyclic basin-floor topography on the depositional evolution of the deep-water Valparaiso forearc basin, central Chile. Basin Research 15: 201-226.

Laursen J, DW Scholl \& R von Huene. 2002. Neotectonic deformation of the central Chile margin: deepwater forearc basin formation in response to hot spot ridge and seamount subduction. Tectonics 21(5): 1-27.

OHI-COI. 2010. GEBCO Gazetteer of Undersea Feature Names. OHI-COI Publication B-8, 409 pp. International Hydrographic Bureau, Monaco.

Rodrigo C. 2001. La tecnología de sonares de 'multihaz' en la Armada de Chile. Anuario Hidrográfico y Oceanográfico de la Armada de Chile 53: 97-104.
Rodrigo C. 2010. Cañones submarinos en el margen continental chileno. En: Díaz-Naveas J \& J Frutos (eds). Geología marina de Chile, 115 pp. Comité Oceanográfico Nacional de Chile, Pontificia Universidad Católica de Valparaíso \& Servicio Nacional de Geología y Minería de Chile, Valparaíso.

Sandwell DT \& WHF Smith. 2001. Bathymetric estimation. En: L Fu \& A Cazenave (eds). Satellite altimetry and Earth sciences. International Geophysical Series 69: 441-457, Academic Press, New York.

SHOA. 2002. 10 años de oceanografía institucional a bordo del AGOR 60 'Vidal Gormaz', 264 pp. Servicio Hidrográfico y Oceanográfico de la Armada, Departamento de Oceanografía, Valparaíso.

Smith WHF. 1993. On the accuracy of digital bathymetric data. Journal of Geophysical Research 98: 9591-9603.

Smith WHF \& DT Sandwell. 1997. Global seafloor topography from satellite altimetry and ship depth soundings. Science 277: 1957-1962.

Smith WHF \& P Wessel. 1990. Gridding with continuous curvature splines in tension. Geophysics 55: 293-305.

Vergara H. 1996. La Cuenca de Valparaíso: antecedentes morfotectónicos y sedimentológicos. Revista de Biología Marina 31(1): 45-63.

Vergara HP \& R Astudillo. 2008. Altos estructurales costa afuera en la región de Valparaíso, Chile central. Revista de Biología Marina y Oceanografía 43(3): 539-545.

Von Huene R, J Corvalán, ER Flueh, J Korstgard \& CONDOR Scientists. 1995. Cruise Report SO101 CONDOR, 171 pp. GEOMAR, Kiel.

Von Huene R, J Corvalán, E Flueh, K Hinz, J Korstgard, C Ranero, W Weinrebe \& CONDOR Scientists. 1997. Tectonic control of the subducting Juan Fernández Ridge on the Andean margin near Valparaiso, Chile. Tectonics 16(3): 474-488.

Wessel P. 1989. Xover: a cross-over error detector for track data. Computers \& Geosciences 15(3): 333-346.

Yáñez GA, CR Ranero, R von Huene \& J Díaz. 2001. Magnetic anomaly interpretation across the southern central Andes $\left(32^{\circ}-34^{\circ} \mathrm{S}\right)$ : the role of the Juan Fernández Ridge in the late Tertiary evolution of the margin. Journal of Geophysical Research 106: 6325-6345.

Recibido el 20 de diciembre de 2010 y aceptado el 09 de marzo de 2011 